\title{
Diagnostic performance of a wearing dynamic ECG recorder for atrial fibrillation screening: the HUAMI heart study
}

Wenxia Fu and Ruogu Li $i^{*}$

\begin{abstract}
Background: Atrial fibrillation (AF) is the most prevalent cardiac dysrhythmia with high morbidity and mortality rate. Evidence shows that in every three patients with AF, one is asymptomatic. The asymptomatic and paroxysmal nature of $A F$ is the reason for unsatisfactory and delayed detection using traditional instruments. Research indicates that wearing a dynamic electrocardiogram (ECG) recorder can guide accurate and safe analysis, interpretation, and distinction of AF from normal sinus rhythm. This is also achievable in an upright position and after exercises, assisted by an artificial intelligence (Al) algorithm.

Methods: This study enrolled 114 participants from the outpatient registry of our institution from June 24, 2020 to July 24, 2020. Participants were tested with a wearable dynamic ECG recorder and 12-lead ECG in a supine, an upright position and after exercises for $60 \mathrm{~s}$.

Results: Of the 114 subjects enrolled in the study, 61 had normal sinus rhythm and 53 had AF. The number of cases that could not be determined by the wristband of dynamic ECG recorder was two, one and one respectively. Case results that were not clinically objective were defined as false-negative or false-positive. Results for diagnostic accuracy, sensitivity, and specificity tested by wearable dynamic ECG recorders in a supine position were $94.74 \%$ (95\% Cl\% 88.76-97.80\%), 88.68\% (95\% Cl 77.06-95.07\%), and 100\% (95\% Cl 92.91-100\%), respectively. Meanwhile, the diagnostic accuracy, sensitivity and specificity in an upright position were $97.37 \%$ (95\% Cl 92.21-99.44\%), 94.34\% (95\% $\mathrm{Cl} 84.03-98.65 \%)$, and 100\% (95\% Cl 92.91-100\%), respectively. Similar results as those of the upright position were obtained after exercise.
\end{abstract}

Conclusion: The widely accessible wearable dynamic ECG recorder integrated with an Al algorithm can efficiently detect $A F$ in different postures and after exercises. As such, this tool holds great promise as a useful and user-friendly screening method for timely AF diagnosis in at-risk individuals.

Keywords: Atrial fibrillation, Detection, Accuracy, Dynamic ECG recorder, One-lead ECG

\section{Background}

Atrial fibrillation (AF) is the most common cardiac dysrhythmia, with an estimated prevalence rate of $2 \%$ and $4 \%$ in adults [1]. Emerging evidence indicates that

\footnotetext{
*Correspondence: liruogu1234@163.com

Department of Cardiac Function, Shanghai Chest Hospital, Shanghai Jiao

Tong University, 241 Huaihai West Rd, Xuhui District, 200030 Shanghai, China
}

the incidence of AF substantially increases with age $(6 \%$; $>65$-years of age and $8-15 \%$; $>80$-years of age) $[2$, 3]. Moreover, findings of the ATRIA study projected a possibility that nearly 3 million individuals aged above 80 -years will be infected with AF by 2050 [4]. AF is related to significant morbidity and mortality, therefore remains a major public health threat [5]. The 2020 ESC guidelines suggested that the lifetime risk of AF 
increased from one in four individuals of European descent to one in three individuals by age 55 [6]. AF is primarily associated with an increased risk of stroke and heart failure, thereby significantly impact people's quality of life and longevity [7, 8]. More evidence shows that AF increases the risk of stroke by approximately five times higher [5] and results in a two-fold risk of heart failure [9].

A previous investigation by Stewart et al. showed that AF constitutes a significant prevalent ratio in the global burden of disease and accounts for $1 \%$ of Britain's National Health Service budget [10]. Elsewhere, Kim et al. revealed that AF contributed significantly to the US Net expenditure. approximated at \$16 billion and $\$ 26$ billion per annum [11]. Emerging data indicate that, of three patients with AF, one is asymptomatic [12]. Hence, a majority of individuals develop heart failure or stroke as the first symptom of AF. Strikingly, asymptomatic AF carries the same risks and sometimes even has more adverse outcomes compared to symptomatic AF [13]. Challenges with the detection of asymptomatic AF in the early stages are becoming an increasing concern. Besides, the European guidelines recommend opportunistic screening for patients with AF over 65-years of age, including high-risk patients [14].

The asymptomatic and paroxysmal nature of AF may result in unsatisfactory and delayed detection using traditional instruments, such as the gold-standard 12-lead electrocardiogram (ECG) [15]. Significantly, ECG is subject to testing space and time limitations and can only be examined in a hospital. In this view, Holter monitoring is associated with prolonged ECG monitoring and makes up for the time limitation caused by 12-lead ECG. Although Holter improves the detection rate of paroxysmal AF, long-term monitoring (24-h and 72-h) influences the patients' daily routine. For example, some patients complain of skin irritation [16].

Notwithstanding cost and other factors, including the inconvenience it causes to patients, large-scale population screening is nearly impossible. The present investigation focused on the patient's resting position without reflecting on the state of the real world. Given these, a rational and user-friendly approach is warranted for early screening and detection of AF in different states. The recent past has seen the increasing use of wristbands as a health management tool. Several individuals wear wristbands daily. Therefore, wearing dynamic ECG recorders will guide the distinction of AF from normal sinus rhythm, accurately and safely in the Chinese population irrespective of postural states.

\section{Methods}

\section{Study population}

A total of 116 outpatients were recruited from the Shanghai Chest Hospital between June 24, 2020 and July 24, 2020. Two participants withdrew informed consent halfway through the study. As such, 114 participants met the inclusion criteria for participation in the study. Participants were aged 18-years and older. Exclusion criteria: (i) Participants who were unable to hold both devices; (ii) participants with severe arterial occlusion or ischemia of the upper limbs; (iii) patients with a pacemaker or implantable cardioverter-defibrillator; (iv) patients who registered for other clinical studies that could impact the purpose of the study. Patients diagnosed as "AF" by 12-lead ECG were classified as AF positive, whereas and AF negative patients served as AF control groups. Standardized in-person interviews per conducted to collect data on baseline characteristics, including demographics, medical history, and medications. A flowchart of the study is shown in Fig. 1.

This is a prospective, registration-only, single-center study whose overall goal is to assess the effectiveness and safety of AF detection and provide a reliable non-invasive method for screening and management of AF in daily routines (ClinicalTrials.govNCT04462653). The local ethics committee of Shanghai Chest Hospital approved this research (No.LS2035), and all participants gave written informed consent before participation in the study. The study was performed following the Helsinki Declaration. The wearable dynamic ECG recorder was approved by the China Food and Drug Administration (Anhui Device Registration Approval No. 20182210012).

\section{Signal acquisition and processing}

Each participant was asked to lie down in a supine position. The choice of an individual's left-or right-hand wristband is pre-set in the wristband's application. Participants held the wearable dynamic ECG recorder on the left wrist on the bed in the ECG room to ensure consistency of results. Of note, during the tests, the attending physician made sure each participant touched the button attached to the 6 o'clock side of the wearable dynamic ECG recorder. After getting tested with a 12-lead ECG for $60 \mathrm{~s}$, subjects actively triggered the wearable dynamic ECG recorder to record the ECG signal for $60 \mathrm{~s}$. Next, participants were asked to wear the wristband in an upright position for $60 \mathrm{~s}$ and after that, they climbed to the third floor and re-tested for $60 \mathrm{~s}$. Finally, a full 12-lead ECG recording for $15 \mathrm{~s}$ was performed immediately to confirm the diagnosis of ECG at this point.

Furthermore, participants were asked to rest their right forefinger on the touch button and press their 


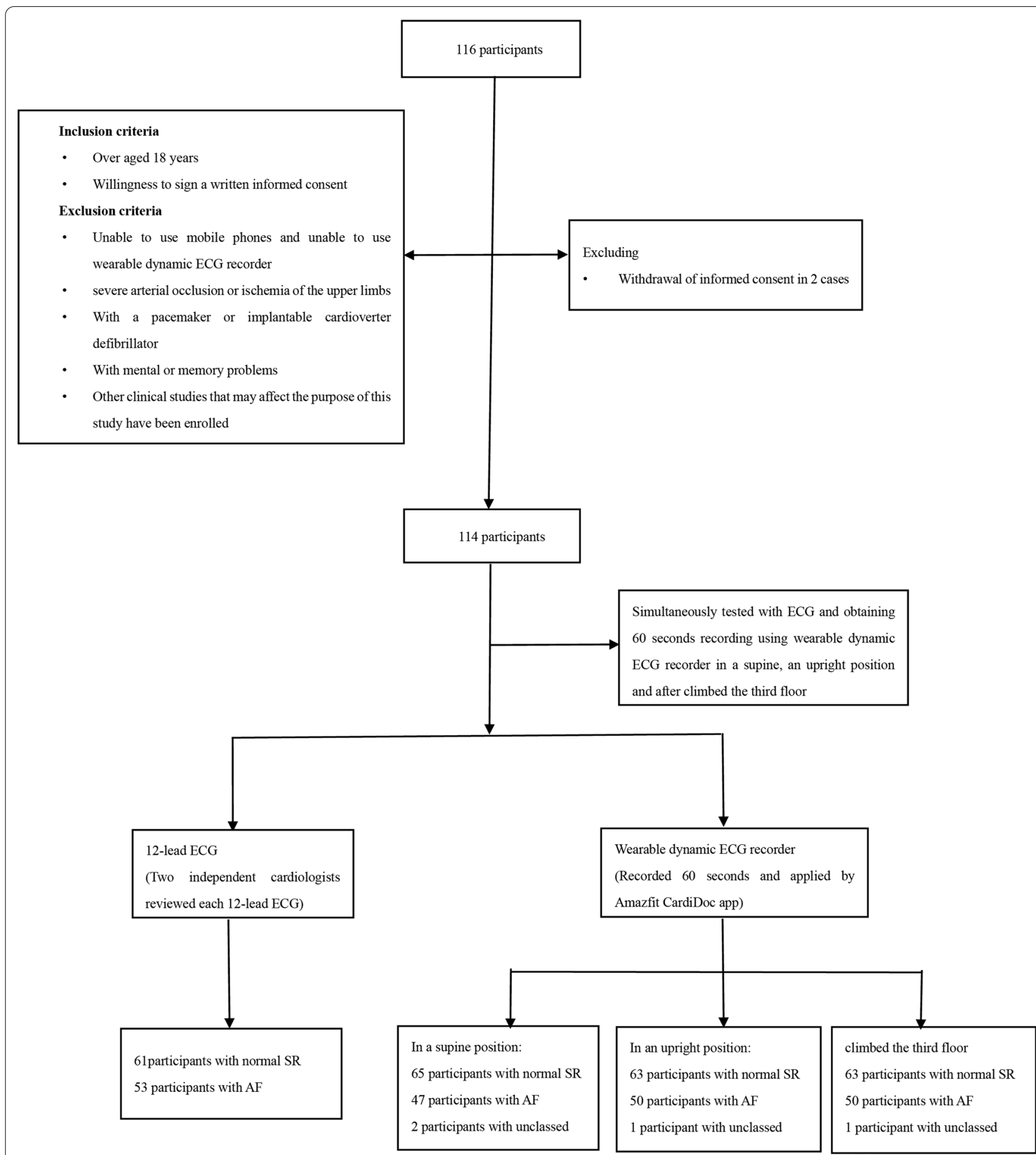

Fig. 1 A flowchart of the study. AF atrial fibrillation, ECG electrocardiogram, SR sinus rhythm

right forefinger using sufficient force to ensure that the contact of electrode sensors with their left wrist's skin for the entire recording duration. Data acquired when a participant wore the wristband on the left hand with the right thumb on the touch button was seen to be equivalent to limb Lead I. The ECG data was transmitted to the Amazfit CardiDoc application and Cloud servers in real-time and analyzed via artificial intelligence (AI) algorithm. 
The device comprised a wristband, ECG recorder, the embedded software (installed on the ECG recorder), mobile phone application (Amazfit CardiDoc application installed on the mobile phone, which transmits the ECG data over the network to the cloud servers), and charging cable, designed to capture the ECG data of the user and transmit it via Bluetooth to an iOS or Android mobile phone. The AmazfitCardiDoc application can store thousands of recordings on the mobile phone. These recordings are accessible to authorized users on cloud servers. The application determines the presence or absence of a classified waveform for AF or sinus rhythm, which is not recommended for other users with known arrhythmias. Figure 2 shows a prototype for AF detection using the wearable dynamic ECG recorder and 12-lead ECG.

\section{Rhythm analysis}

12-lead ECG recordings served as the reference standard for heart rhythm classification. Two independent electrophysiologists who were blinded to the analysis of results reviewed the 12-lead ECG. Possible disagreements were solved by consensus. The software would automatically save the ECG data immediately after the wearable dynamic ECG recorder recordings were complete. The delivered diagnosis was cited by an AI algorithm either as "non-AF," "AF," and "unclassified." If the signal quality was too poor, unstable, or the requirements were not met, the output was "unclassified." The recording stopped if the contact with the electrode was disconnected during the acquisition, therefore was restarted.

\section{Study outcomes}

The primary outcome of the study was the wearable dynamic ECG accuracy of the recorder compared to the 12-lead ECG in detecting AF. The secondary outcomes were the sensitivity, specificity, positive predictive value (PPV), and negative predictive value (NPV) of the wearable dynamic ECG recorder in detecting AF. Safety evaluation criteria included the occurrence of adverse events (AE) and device defects. AE and serious adverse events (SAE) during the clinical study were recorded, and the potential association of $\mathrm{AE}$ and $\mathrm{SAE}$ with the test device was determined. Device defects, including problems associated with the use of test devices, such as failure to wear, no result output, and signal interference were noted.

\section{Statistical methods}

Usually, a sample size of 108 outpatients is required with an $80 \%$ power and an $\alpha$ of 0.05 . Herein, considering a roughly $5 \%$ dropout rate, six outpatients were needed to ensure complete data. Continuous variables with a normal distribution were presented as mean \pm standard deviation (SD). Dates with discrete variables were presented as percentages. Besides, dates with non-normal distribution were presented as medians and interquartile ranges. The estimated $30 \%$ of patients with AF, 54 positive AF samples, and 60 non-AF samples were selected.

Data were analyzed by t-test, Chi-square, or Fisher's exact tests, and the Wilcoxon tests as appropriate. A fourfold table was constructed to diagnose "AF" or "non-AF" using a 12-lead ECG and "AF" or "non-AF" using a wristband algorithm. During AF diagnosis, the matching rate of the difference between the AI algorithm of wristband judgment and human judgment assisted in the evaluation of the consistency of positive results. The number of true positives (TP), true negatives (TN), false positives (FP), and false negative (FN) from the AF and typical sinus rhythm databases were counted.

Subsequently, the sensitivity $\mathrm{TP} /(\mathrm{TP}+\mathrm{FN})$, specificity $\mathrm{TN} /(\mathrm{TN}+\mathrm{FP})$, positive predictive value true positive $\mathrm{TP} /(\mathrm{TP}+\mathrm{FP})$, negative predictive value $\mathrm{TN} /(\mathrm{FN}+\mathrm{TN})$, and accuracy $(\mathrm{TP}+\mathrm{TN}) /(\mathrm{TP}+\mathrm{TN}+\mathrm{FP}+\mathrm{FN})$ were calculated. The "unclassified" cases were defined as the true positive and true negative or false positive and false negative, and then counted and described separately. Statistical analysis was performed by SPSS 22.0 software for Windows (IBM Corp., Armonk, NY, USA). A P-value of $<0.05$ denoted statistical significance.

\section{Results}

\section{Study population and baseline characteristics}

A total of 114 subjects (61 with normal sinus rhythm and 53 with AF), 65 males and 49 females, mean age $59 \pm 11.16$ years (range: $25-80$ years), average height $1.66 \pm 0.08 \mathrm{~m}$ (range: $1.50-1.80 \mathrm{~m}$ ), average weight $66.1 \pm 11.96 \mathrm{~kg}$ (range: 40-105 kg), average body mass index $23.87 \pm 3.28 \mathrm{~kg} / \mathrm{m}^{2}$ (range: $16.65-33.75 \mathrm{~kg} / \mathrm{m}^{2}$ ) were included in the study. Compared to the non-AF group, subjects in the AF group were: (i) significantly older $(P<0.001)$; (ii) had a higher proportion of participants with coronary heart disease $(P=0.014)$; (ii) had a higher thromboembolic risk based on the CHA2DS2VASc $(P=0.001)$; (iv) used oral anticoagulants, antiplatelet agents, calcium channel blockers (CCB), diuretics, digoxin, and $\beta$-blockers at significantly higher rates $($ All $P<0.05)$ (Table 1$)$.

\section{Accuracy and safety evaluation of wearable dynamic ECG recorders in $60 \mathrm{~s}$ in a supine position}

The algorithm automatically determined the $60 \mathrm{~s}$ detection of the wearable dynamic ECG recorder for 47 cases of AF, 65 cases of non-AF, and 2 cases that could be judged (Table 2). The number of cases that were "unable to classify" was defined as "TPTN". The diagnostic accuracy, sensitivity, and specificity using wearable dynamic ECG 


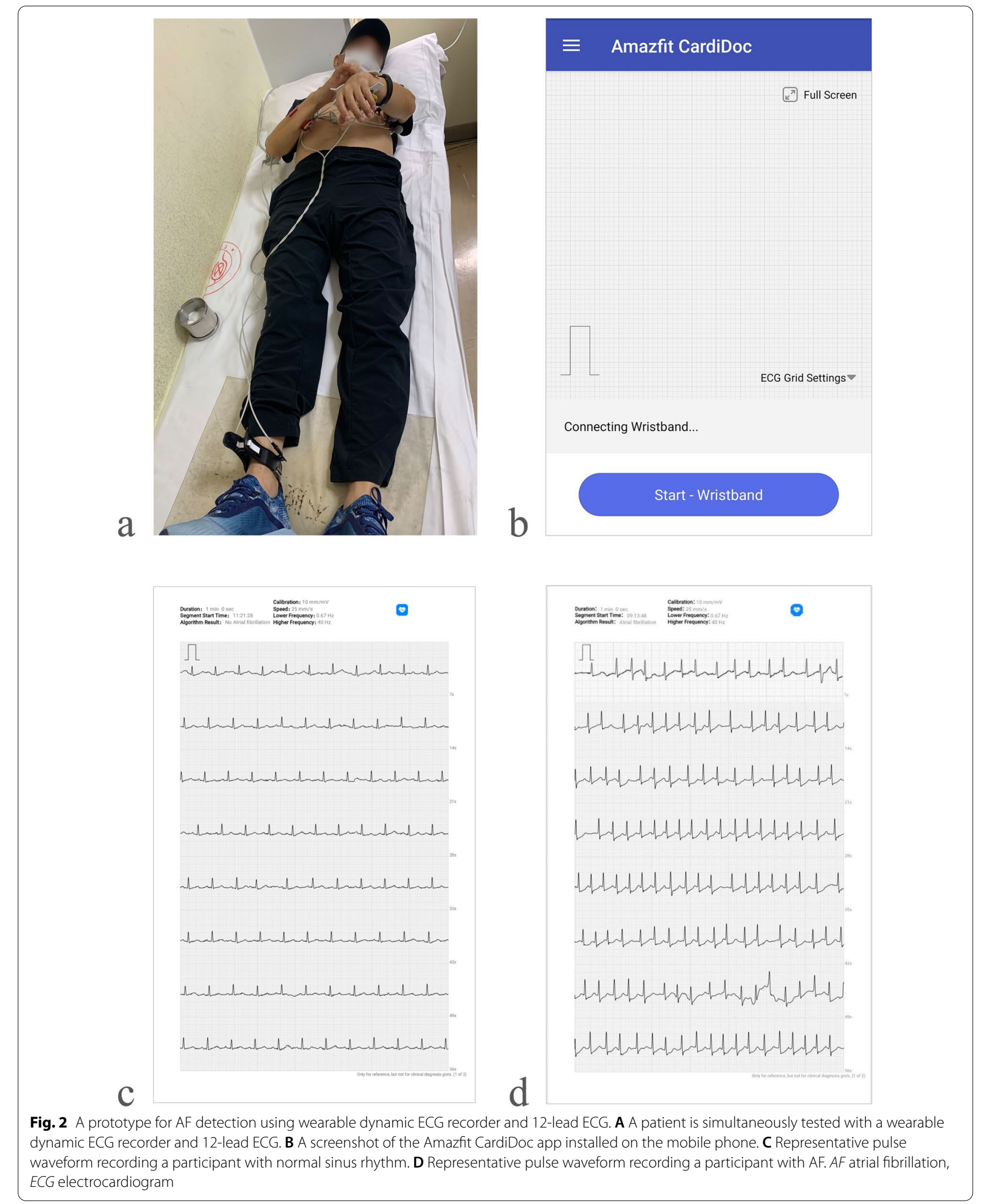


Table 1 Baseline demographic of in Non-AF and AF group $(\mathrm{N}=114)$

\begin{tabular}{|c|c|c|c|}
\hline Variable & Non-AF $(n=61)$ & $A F(n=53)$ & $P$-value \\
\hline \multicolumn{4}{|l|}{ Demographics } \\
\hline Age (years) & $55.15 \pm 11.01$ & $64.00 \pm 9.38$ & $<0.001$ \\
\hline Females (n, \%) & $25(41.0)$ & $24(45.3)$ & 0.644 \\
\hline Height (m) & $1.66 \pm 0.08$ & $1.66 \pm 0.08$ & 0.619 \\
\hline Weight (kg) & $66.16 \pm 11.89$ & $66.07 \pm 12.15$ & 0.965 \\
\hline BMI $\left(\mathrm{kg} / \mathrm{m}^{2}\right)$ & $23.97 \pm 3.21$ & $23.74 \pm 3.38$ & 0.711 \\
\hline \multicolumn{4}{|l|}{ Medical history (n, \%) } \\
\hline Hypertension & $21(34.4)$ & $24(45.3)$ & 0.237 \\
\hline Diabetes mellitus & $6(9.8)$ & $9(17.0)$ & 0.260 \\
\hline Heart failure & $14(23.0)$ & $13(24.5)$ & 0.843 \\
\hline Stroke & $2(3.3)$ & $1(1.9)$ & 0.643 \\
\hline Coronary artery disease & $0(0.0)$ & $5(9.4)$ & 0.014 \\
\hline Vascular disease & $3(4.9)$ & $5(9.4)$ & 0.346 \\
\hline Pulmonary embolism & $0(0.0)$ & $1(1.9)$ & 0.285 \\
\hline Hyperthyroidism & $0(0.0)$ & $1(1.9)$ & 0.285 \\
\hline Renal dysfunction & $0(0.0)$ & $1(1.9)$ & 0.285 \\
\hline Hepatic dysfunction & $1(1.8)$ & $5(9.4)$ & 0.076 \\
\hline Current drinking & $17(27.9)$ & $13(24.5)$ & 0.686 \\
\hline Current smoking & $1(1.6)$ & $2(3.8)$ & 0.090 \\
\hline CHA2DS2-VASc score & $1(1-2.5)$ & $2(1-3)$ & 0.001 \\
\hline \multicolumn{4}{|l|}{ Medication, n (\%) } \\
\hline Oral anticoagulant & $1(1.6)$ & $21(39.6)$ & $<0.001$ \\
\hline Antiplatelet drug & $1(1.6)$ & $11(20.8)$ & 0.001 \\
\hline ACEI/ARBs & $14(23.0)$ & $14(26.4)$ & 0.668 \\
\hline $\mathrm{CCB}$ & $7(11.7)$ & $18(34.0)$ & 0.004 \\
\hline Diuretic & $0(0.0)$ & $8(15.1)$ & 0.002 \\
\hline Digoxin & $0(0.0)$ & $10(19.2)$ & $<0.001$ \\
\hline Beta-blocker & $3(4.9)$ & $28(52.8)$ & $<0.001$ \\
\hline Amiodarone & $1(1.6)$ & $5(9.4)$ & 0.063 \\
\hline
\end{tabular}

$\mathrm{AF}$, atrial fibrillation; $\mathrm{BMI}$, body mass index; $\mathrm{CHA} 2 \mathrm{DS} 2-\mathrm{VASc}$, congestive heart failure, hypertension, age $\geq 75$, diabetes, stroke, vascular disease, age 65-74 and sex category(female); ACEI/ARBs, angiotensin-converting enzyme (ACE) inhibitors, angiotensin-receptor blockers; $C \mathrm{CB}$, calcium channel blocker

Table 2 Testing results of AF detection in participants and overall diagnostic performance of wearable dynamic ECG recorder in $60 \mathrm{~s}$ in a supine position

\begin{tabular}{lrrlr}
\hline Wearable dynamic & \multicolumn{4}{l}{ Standard physician read ECG } \\
\cline { 2 - 5 } ECG recorder & AF & Sinus rhythm & Unclassified & N \\
\hline AF & 47 & 0 & 0 & 47 \\
Non-AF & 4 & 61 & 0 & 65 \\
Unclassified & 2 & 0 & 0 & 2 \\
n & 53 & 61 & 0 & 114 \\
\hline
\end{tabular}

ECG electrocardiogram, $A F$ atrial fibrillation recorders were 96.49\% (95\% CI 91.03-98.92\%), 92.45\% (95\% CI 81.64-97.52\%), and 100\% (95\% CI 92.91-100\%), respectively. The PPV was $100 \%$ (95\% CI 91.32-100\%), while NPV was 93.85\% (95\% CI 84.78-98.02\%) (Table 3).

For cases, such as "unable to classify," which were defined as "FPFN," the diagnostic accuracy, sensitivity, and specificity using wearable dynamic ECG recorders were $94.74 \%$ (95\% CI 88.76-97.80\%), 88.68\% (95\% CI 77.06-95.07\%), and 100\% (95\% CI 92.91-100\%), respectively. The PPV was $100 \%$ (95\% CI 90.98-100\%), and NPV was 91.04\% (95\% CI 81.48-96.16\%) (Table 3).

The medical devices tested in the study belong to category II medical devices with low-risk management levels. No adverse events or obvious device defects occurred within the entire investigation.

\section{Accuracy and safety evaluation of wearable dynamic ECG recorders in $60 \mathrm{~s}$ in an upright position}

The algorithm automatically determined the $60 \mathrm{~s}$ detection of the wearable dynamic ECG recorder for 50 cases of AF, 63 cases of non-AF, and 1 case that could not be judged (Table 4). The number of cases that were "unable to classify" was defined as "TPTN." The diagnostic accuracy, sensitivity, and specificity were 98.25\% (95\% CI\% 93.43-99.91\%), 96.23\% (95\% CI 86.51-99.69\%), and 100\% (95\% CI 92.91-100\%), respectively. The PPV was $100 \%$ (95\% CI 91.63-100\%), while NPV was 96.83\% (95\% CI 88.50-99.77\%) (Table 5).

For cases such as "unable to classify," which were defined as "FPFN," the diagnostic accuracy, sensitivity, and specificity were $97.37 \%$ (95\% CI 92.21-99.44\%), 94.34\% (95\% CI 84.03-98.65\%), and 100\% (95\% CI 92.91$100 \%$ ), respectively. The PPV was $100 \%$ (95\% CI $91.48-$ 100\%), while NPV was 95.31\% (95\% CI 86.57-98.92\%) (Table 5). No adverse events or obvious device defects occurred within the entire investigation.

Accuracy and safety evaluation of wearable dynamic ECG recorders in $60 \mathrm{~s}$ after climbed the third floor

Consistent results with those obtained for the upright position measurement were reported. There were 50 cases of AF, 63 cases of non-AF, and 1case that could not be judged (Table 6). No adverse events or obvious device defects occurred within the entire investigation (Table 7).

\section{Discussion}

The accuracy of wearable dynamic ECG recorder for AF detection from sinus rhythm has herein been demonstrated in a small population trial setting. The finding 
Table 3 Detailed diagnostic performance of the wearable dynamic ECG recorder for AF screening in a supine position

\begin{tabular}{lcc}
\hline & Unable to unclassified as TPTN & Unable to unclassified as FPFN \\
\hline Accuracy, \%(95\% Cl) & $96.49 \%(91.03-98.92 \%)$ & $94.74 \%(88.76-97.80 \%)$ \\
Sensitivity, \%(95\% Cl) & $92.45 \%(81.64-97.52 \%)$ & $88.68 \%(77.06-95.07 \%)$ \\
Specificity, \%(95\% Cl) & $100 \%(92.91-100 \%)$ & $100 \%(92.91-100 \%)$ \\
PPV, \%(95\% Cl) & $100 \%(91.32-100 \%)$ & $100 \%(90.98-100 \%)$ \\
NPV, \%(95\% Cl) & $93.85 \%(84.78-98.02 \%)$ & $91.04 \%(81.48-96.16 \%)$ \\
\hline
\end{tabular}

ECG electrocardiogram, $A F$ atrial fibrillation, $T P$ true positives, $T N$ true negatives, $F P$ false positives, $F N$ false negative, $P P V$ positive predictive value, $N P V$ negative predictive value

Table 4 Testing results of AF detection in participants and overall diagnostic performance of wearable dynamic ECG recorder in $60 \mathrm{~s}$ in an upright position

\begin{tabular}{lrrrr}
\hline Wearable dynamic & \multicolumn{4}{l}{ Standard physician read ECG } \\
\cline { 2 - 5 } & AF & Sinus rhythm & Unclassified & N \\
\hline AF & 50 & 0 & 0 & 50 \\
Non-AF & 2 & 61 & 0 & 63 \\
Unclassified & 1 & 0 & 0 & 1 \\
n & 53 & 61 & 0 & 114 \\
\hline
\end{tabular}

ECG electrocardiogram, $A F$ atrial fibrillation

provides a non-invasive, easy-to-use, and affordable tool to detect and discriminate AF in different positions and after exercises. To the best of our knowledge, the instrument used in this study is the first domestic tool applying an AI algorithm and a single-lead ECG wristband, which is user-friendly, easy to operate, and convenient to wear.

The wearable dynamic ECG recorder with an AI algorithm offers an accurate detection AF in different postures with $100 \%$ specificity and positive predictive value. When subjects are put to stand and tested after exercise, it becomes easier to detect the signal detection and make the correct diagnosis. In the present study, our AI algorithm has been fully trained and tested via large-scale real user data to ensure its reliability. AI is constructed using deep convolutional neural networks. The official data sensitivity and specificity of the test set were $93.36 \%$ and $99.75 \%$, respectively. The single-lead ECG provides physicians with higher specificity and a clear review of the ECG records. At the same time, the wearable dynamic ECG recorder does not impact the daily activities of the subject. Our tested wristband did not require frequent communication with smartphones, consuming less power and increases the time of continuous data recording. Also, the wristband can stand on standby for 7 days on a fully charged battery.

The investigation provides data on the lying position, standing position, and exercise to simulate the test results of the tool in different states. Moreover, once the application detects AF, it promptly sends text messages to the wearer, related relatives, and a designated doctor assigned by Huami. The designated doctor would then diagnose and administer treatment to the patient.

Current evidence indicates that pulse palpation [17], 12-lead ECG [18], 24-h ECG Holter [19] and implanted cardioverter-defibrillator (ICD) [20] methods are some of the traditional methods for AF screening. However, these traditional methods are poised with challenges. With the advancement in technology, the present research has confirmed that smart devices, including mobile phones, handing devices, and wearable devices, can be employed for AF detection. McManus et al. [21] provided an algorithm connecting the root mean square of successive RR difference (RMSSD/mean) and Shannon entropy (ShE). The algorithm demonstrated excellent sensitivity of $96.2 \%$, a specificity of $97.5 \%$, and an accuracy of $96.8 \%$ for the beat-to-beat distinction of

Table 5 Detailed diagnostic performance of the wearable dynamic ECG recorder for AF screening in an upright position

\begin{tabular}{lcc}
\hline & Unable to unclassified as TPTN & Unable to unclassified as FPFN \\
\hline Accuracy, \%(95\% Cl) & $98.25 \%(93.43-99.91 \%)$ & $97.37 \%(92.21 \%, 99.44 \%)$ \\
Sensitivity, \%(95\% Cl) & $96.23 \%(86.51-99.69 \%)$ & $94.34 \%(84.03-98.65 \%)$ \\
Specificity, \%(95\% Cl) & $100 \%(92.91-100 \%)$ & $100 \%(92.91-100 \%)$ \\
PPV, \%(95\% Cl) & $100 \%(91.63-100 \%)$ & $100 \%(91.48-100 \%)$ \\
NPV, \%(95\% Cl) & $96.83 \%(88.50-99.77 \%)$ & $95.31 \%(86.57-98.92 \%)$
\end{tabular}

ECG electrocardiogram, AF atrial fibrillation, $T P$ true positives, $T N$ true negatives, $F P$ false positives, $F N$ false negative, $P P V$ positive predictive value, $N P V$ negative predictive value 
Table 6 Testing results of AF detection in participants and overall diagnostic performance of wearable dynamic ECG recorder in $60 \mathrm{~s}$ after climbed the third floor in an upright position

\begin{tabular}{lrrlr}
\hline Wearable dynamic & \multicolumn{4}{l}{ Standard physician read ECG } \\
\cline { 2 - 5 } ECG recorder & AF & Sinus rhythm & Unclassified & N \\
\hline AF & 50 & 0 & 0 & 50 \\
Non-AF & 2 & 61 & 0 & 63 \\
Unclassified & 1 & 0 & 0 & 1 \\
$\mathrm{n}$ & 53 & 61 & 0 & 114 \\
\hline ECG electrocardiogram, $A F$ atrial fibrillation & &
\end{tabular}

an irregular pulse during AF from sinus rhythm. Elsewhere, Svennberget et al. [22] described a handheld ECG recorder for intermittent ECG recordings, integrated with a mobile transmitter that sends 30-s ECG strip data to a database. In their analysis, repeated routine ECG examination over a long period potentially improved AF detection sensitivity to four times the number of cases diagnosed via routine ECG examination at a single time point.

The AF-SCREEN international collaboration [23], in 2017, confirmed the advantages of handheld ECG devices, particularly in providing a confirmable ECG trace required by the diagnostic guidelines for AF. Another mSToPS randomized clinical trial by Steinhubl et al. [24] provided evidence on the impact of a selfapplied wearable ECG patch in AF detection. Further analysis demonstrated a higher new diagnosis rate of AF in the immediate group at 4 months than that of the delayed group (3.9\% vs. $0.9 \%$, the absolute difference was $3.0 \%$, 95\% CI $1.8-4.1 \%$ ).

A recent technology of photo-plethysmography (PPG) application was mentioned for the detection of AF. The sensitivity and specificity of the PPG algorithm for AF detection were $97-100 \%$ and $92-94 \%$, respectively [25]. Furthermore, conclusions of HeartStudy-MAFA II [26] showed higher sensitivity and specificity of the PPG method in AF screening. The sensitivity, specificity, and a positive predictive value achieved with their method were $100 \%, 99 \%$, and $91.6 \%$, respectively. Their data were collected in a supine position, therefore, does not reflect the actual situation for home screening in which the movement has a more significant influence on the PPG signal. Of note, interference caused by movement should be avoided between single-lead ECG and the PPG technology to improve accuracy. Also, there is a tendency to use single-lead ECG recordings in several patients with paroxysmal AF since single-lead ECG recordings with mobile phones or wristbands for AF detection are clinically superior to PPG signals.

Emerging data shows that early intervention and qualifiable risk factor control can reduce the incidence of AF. In this view, a wearing dynamic ECG recorder may provide an essential tool for patients with or at risk to detect AF. This approach allows timely detection and management of AF before adverse health consequences such as ischemic stroke or heart failure occur. With the increase of health awareness, wearable monitoring technology is gaining attention in health, allowing patients to comfortably manage symptoms from their places of residence. The wearable dynamic ECG recorder is a feasible and accurate tool that allows users to monitor and track their ECG recordings and share them with the attending physicians.

There are a few limitations to the study. Firstly, the ECG monitoring was performed in asymptomatic participants and not in subjects with symptoms. Secondly, the instruments were only used for discriminating between AF and sinus rhythm yet the wearable dynamic ECG recorder could detect other arrhythmias, including premature beats, atrial tachyarrhythmias, and atrial flutter. In our future study, new algorithms will be integrated to assist the identification and distinction between sinus arrhythmias and various arrhythmia forms. Lastly, owing to the sample size was relatively small, more extensive testing would be warranted in the future to verify the results.

Table 7 Detailed diagnostic performance of the wearable dynamic ECG recorder for AF screening after climbed the third floor in an upright position

\begin{tabular}{lcc}
\hline & Unable to unclassified as TPTN & Unable to unclassified as FPFN \\
\hline Accuracy, \%(95\% Cl) & $98.25 \%(93.43-99.91 \%)$ & $97.37 \%(92.21 \%, 99.44 \%)$ \\
Sensitivity, \%(95\% Cl) & $96.23 \%(86.51-99.69 \%)$ & $94.34 \%(84.03-98.65 \%)$ \\
Specificity, \%(95\% Cl) & $100 \%(92.91-100 \%)$ & $100 \%(92.91-100 \%)$ \\
PPV, \%(95\% Cl) & $100 \%(91.63-100 \%)$ & $100 \%(91.48-100 \%)$ \\
NPV, \%(95\% Cl) & $96.83 \%(88.50-99.77 \%)$ & $95.31 \%(86.57-98.92 \%)$ \\
\hline
\end{tabular}

ECG electrocardiogram, AF atrial fibrillation, $T P$ true positives, $T N$ true negatives, $F P$ false positives, $F N$ false negative, $P P V$ positive predictive value, $N P V$ negative predictive value 


\section{Conclusions}

The broadly accessible wearable dynamic ECG recorder with an AI algorithm can detect AF and analyze heart rhythms at different postures and after exercises. This approach may provide a user-friendly and accurate method to discriminate AF and timely detect asymptomatic AF patients or those at risk of AF. More extensive clinical trials are needed to assess the effectiveness of this technique in monitoring and early diagnosis of AF recurrence.

\begin{abstract}
Abbreviations
AF: Atrial fibrillation; ECG: Electrocardiogram; Al: Artificial intelligence; PPV: Positive predictive value; NPV: Negative predictive value; AE: Adverse events; SAE: Serious adverse events; SD: Standard deviation; TP: True positives; TN: True negatives; FP: False positives; FN: False negative; CCB: Calcium channel blockers; ICD: Implanted cardioverter-defibrillator; She: Shannon entropy; PPG: Photo-plethysmography.
\end{abstract}

\section{Acknowledgements}

The authors declare no conflict of interest.

\section{Authors' contributions}

WXF took responsibility for all aspects of the reliability and freedom from bias of the data presented and was a major contributor in writing the manuscript. RGL took responsibility for research design. Both the authors read and approved the final manuscript.

\section{Funding}

Not applicable.

\section{Availability of data and materials}

Datasets used and/or analyzed in this study are de-identified and available from the corresponding author on reasonable request.

\section{Declarations}

\section{Ethics approval and consent to participate}

The study was approved by the Shanghai Chest Hospital's local ethics committee. Written informed consent from all participants was obtained.

\section{Consent for publication}

Not applicable.

\section{Competing interests}

The authors declare that they have no competing interests.

Received: 23 December 2020 Accepted: 3 November 2021

Published online: 20 November 2021

\section{References}

1. Benjamin EJ, Muntner P, Alonso A, Bittencourt MS, Callaway CW, Carson AP, et al. Heart disease and stroke statistics-2019 update: a report from the American Heart Association. Circulation. 2019;139:e56-528. https:// doi.org/10.1161/CIR.0000000000000659.

2. Davis RC, Hobbs FD, Kenkre JE, Roalfe AK, lles R, Lip GY, et al. Prevalence of atrial fibrillation in the general population and in high-risk groups: the ECHOES study. Europace. 2012;14:1553-9. https://doi.org/10.1093/europ ace/eus087.

3. Benjamin EJ, Levy D, Vaziri SM, D'Agostino RB, Belanger AJ, Wolf PA, et al. Independent risk factors for atrial fibrillation in a population-based cohort. The Framingham Heart Study. JAMA. 1994;271:840-4.
4. Go AS, Hylek EM, Phillips KA, Chang Y, Henault LE, Selby JV, et al. Prevalence of diagnosed atrial fibrillation in adults: national implications for rhythm management and stroke prevention: the AnTicoagulation and Risk Factors in Atrial Fibrillation (ATRIA) Study. JAMA. 2001;285:2370-5. https://doi.org/10.1001/JAMA.285.18.2370.

5. Lowres N, Redfern J, Freedman SB, Orchard J, Bennett AA, Briffa T, Bauman A, Neubeck L, et al. Choice of Health Options In prevention of Cardiovascular Events for people with Atrial Fibrillation (CHOICE-AF): a pilot study. Eur J Cardiovasc Nurs. 2016;15:39-46. https://doi.org/10.1177/14745 15114549687

6. Hindricks G, Potpara T, Dagres N, Arbelo E, Bax JJ, Blomström-Lundqvist C, et al. 2020 ESC Guidelines for the diagnosis and management of atrial fibrillation developed in collaboration with the European Association of Cardio-Thoracic Surgery (EACTS). Eur Heart J. 2020;29:ehaa612. https:// doi.org/10.1093/eurheartj/ehaa61.

7. Tsang TS, Petty GW, Barnes ME, O'Fallon WM, Bailey KR, Wiebers DO, et al. The prevalence of atrial fibrillation in incident stroke cases and matched population controls in Rochester, Minnesota: changes over three decades. J Am Coll Cardiol. 2003;42:93-100. https://doi.org/10.1016/s07351097(03)00500-x.

8. Wang TJ, Wang TJ, Larson MG, Levy D, Vasan RS, Leip EP, Wolf PA, et al. Temporal relations of atrial fibrillation and congestive heart failure and their joint influence on mortality: the Framingham Heart Study. Circulation. 2003;107:2920-5. https://doi.org/10.1161/01.Cir.0000072767.89944. 6 .

9. Stewart S, Hart CL, Hole DJ, McMurray JJ. A population-based study of the long-term risks associated with atrial fibrillation: 20-year follow-up of the Renfrew/Paisley study. Am J Med. 2002;113:359-64. https://doi.org/10. 1016/s0002-9343(02)01236-6.

10. Stewart S, Murphy NF, Walker A, McGuire A, McMurray JJ. Cost of an emerging epidemic: an economic analysis of atrial fibrillation in the UK. Heart. 2004;90:286-92. https://doi.org/10.1136/hrt.2002.008748.

11. Kim MH, Johnston SS, Chu BC, Dalal MR, Schulman KL. Estimation of total incremental health care costs in patients with atrial fibrillation in the United States. Circ Cardiovasc Qual Outcomes. 2011;4:313-20. https://doi. org/10.1161/circoutcomes.110.958165.

12. Lip GY, Lane DA. Stroke prevention in atrial fibrillation: a systematic review. JAMA. 2015;313:1950-62. https://doi.org/10.1001/JAMA.2015. 4369.

13. Camm AJ, Camm AJ, Kirchhof P, Lip GY, Schotten U, Savelieva I, Ernst S, et al. Guidelines for the management of atrial fibrillation: the Task Force for the Management of Atrial Fibrillation of the European Society of Cardiology (ESC). Eur Heart J. 2010;31:2369-429. https://doi.org/10.1093/ eurheartj/ehq278.

14. Kirchhof P, Benussi S, Kotecha D, Ahlsson A, Atar D, Casadei B, et al. 2016 ESC guidelines for the management of atrial fibrillation developed in collaboration with EACTS. Eur Heart J. 2016;37:2893-962. https://doi.org/ 10.1093/eurheartj/ehw210.

15. Camm AJ, Corbucci G, Padeletti L. Usefulness of continuous electrocardiographic monitoring for atrial fibrillation. Am J Cardiol. 2012;110:270-6. https://doi.org/10.1016/j.amjcard.2012.03.021.

16. Tu HT, Spence S, Kalman JM, Davis SM. Twenty-eight day Holter monitoring is poorly tolerated and insensitive for paroxysmal atrial fibrillation detection in cryptogenic stroke. Intern Med J. 2014;44:505-8. https://doi. org/10.1111/imj.12410.

17. Jaakkola J, Vasankari T, Virtanen R, Juhani Airaksinen KE. Reliability of pulse palpation in the detection of atrial fibrillation in an elderly population. Scand J Prim Health Care. 2017;35:293-8. https://doi.org/10.1080/02813 432.2017.1358858.

18. Jonas DE, Kahwati LC, Yun JDY, Middleton JC, Coker-Schwimmer M, Asher GN. Screening for atrial fibrillation with electrocardiography: evidence report and systematic review for the US Preventive Services Task Force. JAMA. 2018;320:485-98. https://doi.org/10.1001/JAMA.2018.4190.

19. Sanak D, Hutyra M, Kral M, Bartkova A, Zapletalova J, Fedorco M, et al. Paroxysmal atrial fibrillation in young cryptogenic ischemic stroke: a 3-week ECG Holter monitoring study. Biomed Pap Med Fac Univ Palacky Olomouc Czech Repub. 2015;159:283-7. https://doi.org/10.5507/bp.2015. 019.

20. Deshmukh A, Brown ML, Higgins E, Schousek B, Abeyratne A, Rovaris G, et al. Performance of atrial fibrillation detection in a new single-chamber 
ICD. Pacing Clin Electrophysiol. 2016;39:1031-7. https://doi.org/10.1111/ pace.12918.

21. McManus DD, Lee J, Maitas O, Esa N, Pidikiti R, Carlucci A, et al. A novel application for the detection of an irregular pulse using an iPhone $4 \mathrm{~S}$ in patients with atrial fibrillation. Heart Rhythm. 2013;10:315-9. https://doi. org/10.1016/j.hrthm.2012.12.001.

22. Svennberg E, Engdahl J, Al-Khalili F, Friberg L, Frykman V, Rosenqvist M, et al. Mass screening for untreated atrial fibrillation: the STROKESTOP study. Circulation. 2015;131:2176-84. https://doi.org/10.1161/circulatio naha.114.014343.

23. Freedman B, Camm J, Calkins H, Healey JS, Rosenqvist M, Wang J, et al. Screening for atrial fibrillation: a report of the AF-SCREEN international collaboration. Circulation. 2017;135:1851-67. https://doi.org/10.1161/ circulationaha.116.026693.

24. Steinhubl SR, Waalen J, Edwards AM, Ariniello LM, Mehta RR, Ebner GS, et al. Effect of a home-based wearable continuous ECG monitoring patch on detection of undiagnosed atrial fibrillation: the MSToPS randomized clinical trial. JAMA. 2018;320:146-55. https://doi.org/10.1001/jama.2018. 8102.

25. Kotecha D, Breithardt G, Camm AJ, Lip GYH, Schotten U, Ahlsson A, et al. Integrating new approaches to atrial fibrillation management: the 6th AFNET/EHRA Consensus Conference. Europace. 2018;20:395-407. https:// doi.org/10.1093/europace/eux318.

26. Zhang H, Zhang J, Li HB, Chen YX, Yang B, Guo YT, et al. Validation of single centre pre-mobile atrial fibrillation apps for continuous monitoring of atrial fibrillation in a real-world setting: pilot cohort study. J Med Internet Res. 2019;21:e14909. https://doi.org/10.2196/14909.

\section{Publisher's Note}

Springer Nature remains neutral with regard to jurisdictional claims in published maps and institutional affiliations.
Ready to submit your research? Choose BMC and benefit from:

- fast, convenient online submission

- thorough peer review by experienced researchers in your field

- rapid publication on acceptance

- support for research data, including large and complex data types

- gold Open Access which fosters wider collaboration and increased citations

- maximum visibility for your research: over $100 \mathrm{M}$ website views per year

At BMC, research is always in progress.

Learn more biomedcentral.com/submissions 